\title{
Der historische Buchbestand der Universitätssternwarte Wien
}

\author{
Karin Lackner, Isolde Müller, Franz Kerschbaum, \\ Roland Ottensamer und Thomas Posch \\ Insitut für Astronomie der Universität Wien, \\ Türkenschanzstraße 17, A-1180 Wien, Austria
}

\begin{abstract}
The Vienna University Observatory houses one of the most important collections of historically significant science books reaching from the $15^{\text {th }}$ to the $18^{\text {th }}$ century. Especially within the Central European countries no other astronomical library with a comparable, historically grown fund exists.

The first Vienna University Observatory was founded in 1755 and Jesuit Maximilian Hell (1720-1792) was nominated as its first director. Therefore the inventory of the Astronomy Library, as well as the inventory of the Vienna University Library, mainly arose from the Jesuit's collections. The Institute of Astronomy Library contains 5 books older than 1500, 56 printed before 1600 and a total of 500 books prior to 1800 - which form the Rare Book Collection.

Two printed catalogues with all the books in the Rare Book Collection are now available. Every entry is illustrated and key data to every book are listed. Important works are annotated with detailed information on their history.
\end{abstract}

\section{Einleitung}

Die Fachbereichsbibliothek Astronomie der Universität Wien verfügt über eine mehr als 500 Titel umfassende Sammlung historischer Druckschriften aus dem 15.-18. Jahrhundert. Die etwa 200 aus dem 15.-17. Jahrhundert stammenden Bücher wurden bereits in ,,Der historische Buchbestand der Universitätssternwarte Wien. Ein illustrierter Katalog. Teil 1" dokumentiert. Im 2006 erschienenen zweiten Teil werden die insgesamt 317 Titel aus dem 18. Jahrhundert präsentiert. 

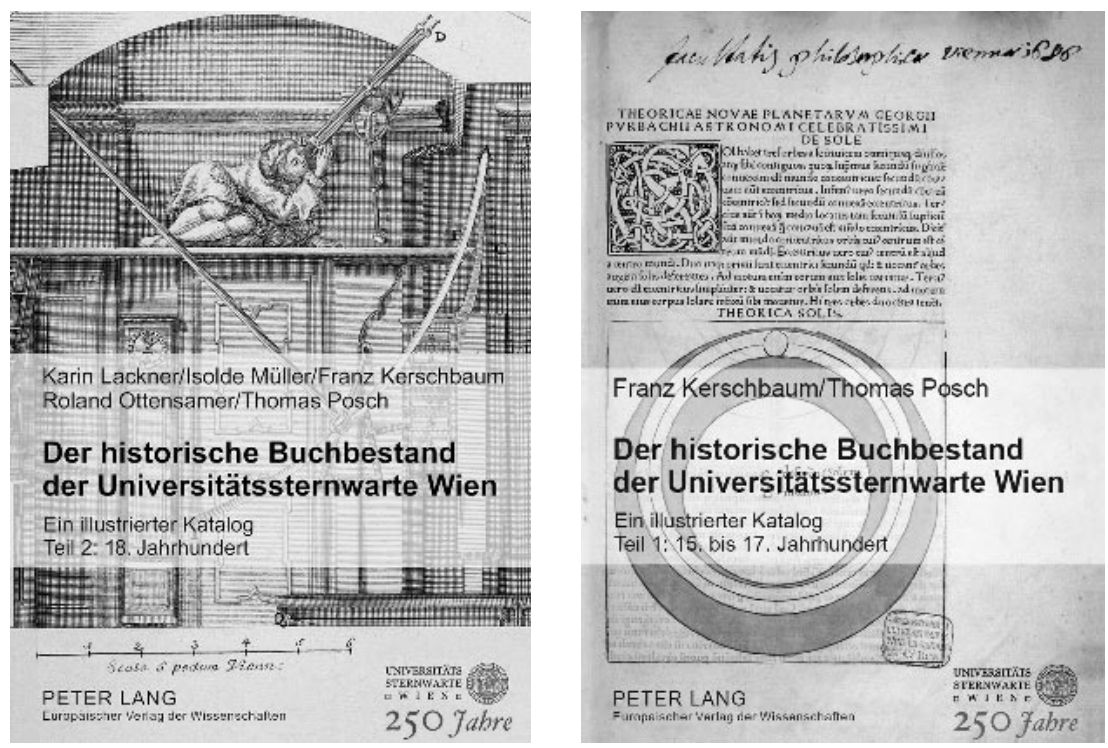

Abb. 1: Linkes Bild: Titelseite Band 1 - Rechtes Bild: Titelseite Band 2

Ein solcher Katalog, in welchem die Bücher dargestellt werden sollten, wurde bereits im ,,Adreßbuch der Bibliotheken der Oesterreichisch-ungarischen Monarchie" "1 angekündigt, dieses lange uneingelöste Versprechen wurde nun mit Erscheinen der beiden Bände über den historischen Buchbestand erfüllt.

Über eine Präsentation des Bestandes aus dem 18. Jahrhundert in Buchform hinauszugehen, ist nicht geplant - unter anderem deswegen nicht, weil im 19. Jahrhundert die industrielle Buchproduktion einsetzte, was die Anzahl der Neuerscheinungen stark in die Höhe trieb und den Stellenwert des jeweiligen Einzeltitels tendenziell reduzierte. Die unter anderem durch die Industrialisierung der Buchproduktion ermöglichte Zunahme der Neuerscheinungen pro Jahr spiegelt sich auch in der Bestandsgeschichte der Wiener Universitätssternwarte wider: Im 19. Jahrhundert kommen rund 2500 Titel neu hinzu, was beinahe einer Verzehnfachung der Zuwachsrate entspricht und eine ebenso ausführliche Dokumentation für diese neueren Bücher schwer realisierbar erscheinen lässt.

\footnotetext{
${ }^{1}$ Johann Bohatta, Michael Holzmann, Adreßbuch der Bibliotheken der Oesterreichisch-ungarischen Monarchie (Wien 1900), S.338
} 


\section{Elektronische Inventarisierung des Buchbestandes aus den Jahren 1700-1799}

Bei der elektronischen Erfassung der Werke aus dem 18. Jahrhundert wurde, wie bereits für jene aus dem 15.-17. Jahrhundert, nach alten Inventarlisten vorgegangen. Hierbei wurden einerseits in diesen Listen nicht aufscheinende Bücher entdeckt, andererseits konnten manche in den Listen geführten Bücher nicht mehr gefunden werden. Dabei handelt es sich teilweise um Dubletten, die vermutlich in den 1930er-Jahren aufgrund von Geldmangel infolge der allgemein schlechten wirtschaftlichen Lage für wichtige Neuanschaffungen verkauft worden sind.

Im Zuge der Inventarisierung wurden zunächst grundlegende Schlüsseldaten erfasst; zu diesen zählen neben dem Titel auch Autor sowie Erscheinungsjahr und -ort, darüber hinaus wurden Angaben wie Sprache, Auflagenbezeichnung, Verlag bzw. Drucker, Umfang, Format, Bemerkungen zu Besonderheiten des jeweiligen Werkes, bibliographische Nachweise und gegebenenfalls beigefügte Werke festgehalten. Für die spätere Einleitung konservatorischer Maßnahmen, sofern erforderlich, war es zusätzlich notwendig, den Erhaltungszustand jedes einzelnen Buches zu dokumentieren. Um globale elektronische Recherchen zu vereinfachen und einen möglichst originalgetreuen Eindruck der Bücher zu vermitteln, wurden über 800 digitale Fotografien, die sowohl Titelseiten aller Werke als auch Schlüsselseiten sowie informative Illustrationen umfassen und über den Link http://www.ub.univie.ac.at/fb-astronomie abrufbar sind, angefertigt.

\section{Buchbeschreibung}

In der Einleitung wird zunächst ein Abriss der Wissenschaftsgeschichte und besonders der Astronomie des 18. Jahrhunderts anhand einiger Schwerpunkte, die sich auch im Buchbestand der Bibliothek der Wiener Universitätssternwarte widerspiegeln, gegeben. Solche Schwerpunkte sind u.a. Gestalt der Erde, Venusdurchgänge und die Entwicklungen im Bereich Sonnensystem, Milchstraße und Kosmos.

Auch zwei herausragende Persönlichkeiten der astronomischen Forschung in Wien - Johann Jakob von Marinoni und Maximilian Hell - werden vorgestellt. Weiters wird auf einige Grundzüge der Geschichte des Buchwesens im 18. Jahrhundert, das Aufkommen von Periodika sowie die fachliche Aufgliederung des Buchbestandes der Wiener Universitätssternwarte eingegangen.

Auf den Katalogteil folgen die Kommentare zu einigen ausgewählten Werken. Auswahlkriterien waren unter anderem der Bezug des jeweiligen Werkes bzw. Autors zu Wien, der Bekanntheitsgrad des jeweiligen Verfassers - Bücher bekannter Autoren wurden bevorzugt kommentiert - sowie die Inno- 

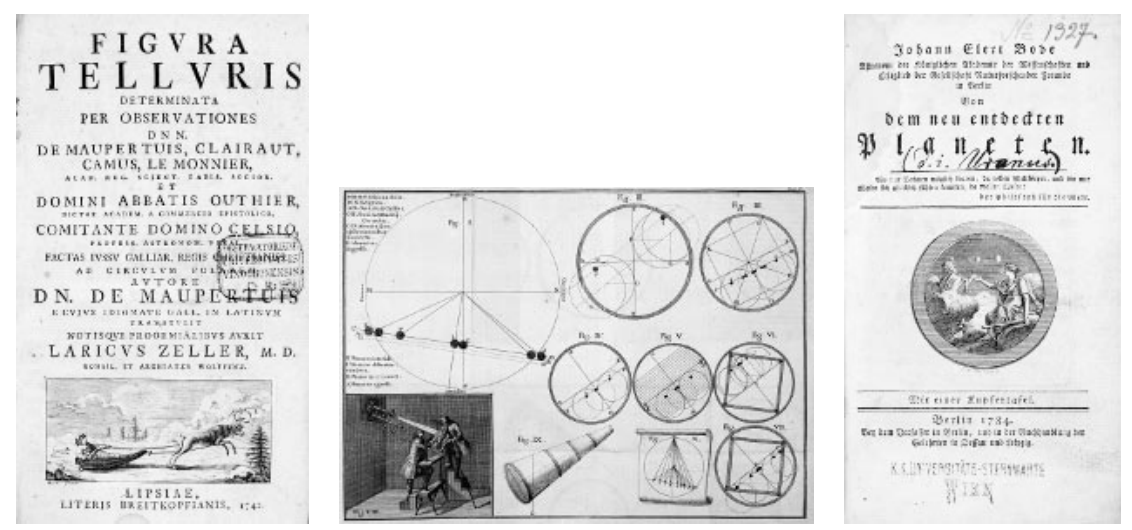

Abb. 2: Links: Maximilian Hell, Transitus Veneris per discum Solis anni 1761 (1761), Illustration zum Venusdurchgang - Mitte: Pierre Louis Moreau de Maupertuis, Figura Telluris (1742), Titelseite - Rechts: Johann Elert Bode, Von dem neu entdeckten Planeten (1784), Titelseite

vativität eines Titels in Bezug auf die Entwicklung neuer Fragestellungen der Forschung (z.B. Erdgestalt).

\section{Buchbestand}

Der regen Sammlertätigkeit der Jesuiten verdankt die heutige UniversitätsSternwarte einen Großteil ihrer wertvollsten Bücher. Für das 18. Jahrhundert - alle jene Bücher, deren Erscheinungsjahr zwischen 1700 und 1799 liegt ergibt sich eine Gesamtzahl von 317 Titeln, wobei mehrbändige Werke als ein Titel gelten; jedoch werden spätere Auflagen früherer Werke - wie zum Beispiel Newtons Philosophiae Naturalis Principia Mathematica aus 1713, 1739 und 1783 - einzeln gezählt.

Insgesamt liegen 117 Werke auf Latein vor, 114 auf Deutsch, 64 auf Französisch. Der restliche Bestand teilt sich auf neun englische, zwei niederländische, zwei spanische, ein italienisches, ein polnisches und sieben mehrsprachige ${ }^{2}$ Werke auf. In der ersten Hälfte des 18. Jahrhunderts, also 1700 bis einschließlich 1749, überwiegen lateinische Werke (56), in etwa gleichem Ausmaß

\footnotetext{
${ }^{2}$ Als mehrsprachige Werke werden jene, in denen die verschiedenen Sprachen in etwa gleichem Ausmaß vertreten sind, bezeichnet. Es gibt vier deutsch-lateinische, einen englisch-lateinischen, einen deutsch-französischen und einen lateinisch-französischen Titel.
} 
folgen französische (22) und deutsche (18). In der zweiten Hälfte, 1750 bis einschließlich 1799, sind deutsche Werke (96) häufiger vorhanden als lateinische (61) oder französische (42). Diese Aufschlüsselung repräsentiert gewissermaßen den im 18. Jahrhundert vor sich gehenden Wandel, dass im Zuge der Aufklärung Latein als Wissenschaftssprache in den Hintergrund trat und die Autoren vermehrt in der jeweiligen Nationalsprache publizierten.

Die Werke des 18. Jahrhunderts lassen sich in folgende Sachgebiete unterteilen: Astronomie einschließlich Himmelsatlanten und Instrumentenkunde (150 Werke, dies entspricht rund 46\% des Gesamtbestandes), Mathematik (66), Physik (27), Biographien (8), Geodäsie (7), Geschichte (6), Chronologie (4), Geographie einschließlich Lexika (4), Meteorologie (2). Die übrigen 43 Werke lassen sich nicht eindeutig einem der oben erwähnten Sachgebiete zuordnen, wie zum Beispiel Christiaan Huygens Opera varia (1724) und Leonhard Eulers Lettres à une princesse d'Allemagne (1768-72).

Vieles mehr lässt sich den beiden im Peter Lang Verlag erschienen Katalogbänden entnehmen:

Der historische Buchbestand der Universitätssternwarte Wien. Ein illustrierter Katalog, Teil 1: 15. bis 17. Jahrhundert

von F. Kerschbaum, Th. Posch (2005)

ISBN 978-3-631-52890-7

Der historische Buchbestand der Universitätssternwarte Wien. Ein illustrierter Katalog, Teil 2: 18. Jahrhundert

von K. Lackner, I. Müller, F. Kerschbaum, R. Ottensamer, Th. Posch (2006) ISBN 978-3-631-53868-5

\section{Danksagungen}

Das Projekt wurde dankenswerter Weise unterstützt vom Bundesministerium für Bildung, Wissenschaft, und Kultur, der Wissenschafts- und Forschungsförderung der Stadt Wien, MA 7 und der Österreichischen Akademie der Wissenschaften. 